\title{
Evaluation of serum levels in T3, T4 and TSH in beta-thalassemic patients referred to the Abuzar hospital in Ahwaz
}

Zari Tahannejad Asad ${ }^{1}$, Majid Ghazanfari ${ }^{1,2}$, Seyyed Nima Naleini ${ }^{3}$, Azam Sabagh ${ }^{1,2}$, Wesam Kooti ${ }^{3}$

${ }^{1}$ Department of Laboratory Sciences, School of Paramedicine, Health Research Institute, Research Center of Thalassemia \& Hemoglobinopathy, Ahvaz Jundishapur University of Medical Sciences, Ahvaz, Iran

${ }^{2}$ Student Research Committee, Ahvaz Jundishapur University of Medical Sciences, Ahvaz, Iran

${ }^{3}$ Student Research Committee, Kurdistan University of Medical Sciences, Sanandaj, Iran

\section{Type of article: Original}

\begin{abstract}
Introduction: Regarding the functioning of the endocrine system, and especially in the thyroid of patients with thalassemia, multiple studies in different parts of the world have reported conflicting results. The aim of this study was to assess the levels of thyroid hormones and TSH in beta-thalassemic patients in the city of Ahwaz.

Methods: In this matched case-control study, 105 patients in the case group and 105 subjects as controls were randomly selected from clients referred to the Abuzar hospital in 2015-2016. Serum levels of T3, T4, and TSH hormones were measured using ELISA. Data was processed with the SPSS15 software and tested by using independent $\mathrm{t}$-tests and logistic regression.

Results: The study results showed that the serum level of $\mathrm{T} 3$ hormone did not significantly differ between the two groups ( $p>0.05)$. Whereas the serum level of T4 was lower in the case group, compared to the controls, which was statistically significant $(\mathrm{p}<0.05)$. The serum level of thyroid stimulating hormone (TSH) in the case group was higher than the control group, and this difference was statistically significant $(p<0.05)$. Logistic regression analysis showed significant differences in serum levels of T4 (OR: 0.58) and TSH (OR: 1.57) between the case and control groups $(\mathrm{p}<0.05)$.

Conclusion: Hypothyroidism is clear in patients with beta thalassemia. With regard to the possible effect of iron on the disorder, a blood transfusion schedule should be performed at intervals, and the desferal injection program should be done regularly to prevent hemochromatosis in patients with thalassemia major due to the effect of accumulation of iron on thyroid function and detection of hypothyroidism. This course of action will prevent incidence of this complication in patients with thalassemia major.
\end{abstract}

Keywords: Thyroid, Beta-thalassemia, Ahwaz

\section{Introduction}

Thalassemia is a genetic disease with an autosomal recessive inheritance pattern in which the generation of one or more hemoglobin chains have been dropped or completely inhibited. Patients with thalassemia major need regular monthly blood transfusions starting at an age of approximately 6 months to maintain oxygen supply to tissues due to severe anemia. Followed by ineffective hematopoiesis, patients with thalassemia often have high iron absorption from the gastrointestinal tract, and despite iron overload, continue to have a greater intestinal absorption. On the other hand, regular blood transfusions cause iron overload in patient's organs. Through the formation of free radicals and lipid peroxidation, iron overload causes damage to transferrin receptors on the cell surface and some cellular organelles, which disrupts their tolerance and viability, compared to non-organic iron. When levels of total body iron reach $40 \mathrm{~g}$, the function of body organs are disrupted, and at the level of $60 \mathrm{~g}$ or more, irreversible heart failure occurs. The major cause of mortality and morbidity in these patients is iron overload in parenchymal tissues. Most patients with thalassemia major will die between ages 16 and 24 due to iron overload, and almost all deaths occur

\section{Corresponding author:}

Wesam Kooti, Kurdistan University of Medical Sciences, Sanandaj, Iran.

Tel: +98. 9336583929, Email: wesamkooti@gmail.com

Received: March 16, 2016, Accepted: May 14, 2016, Published: July 2016

iThenticate screening: May 14, 2016, English editing: June 26, 2016, Quality control: July 04, 2016

(C) 2016 The Authors. This is an open access article under the terms of the Creative Commons Attribution-NonCommercialNoDerivs License, which permits use and distribution in any medium, provided the original work is properly cited, the use is non-commercial and no modifications or adaptations are made. 
due to iron overload in the heart muscle. The chronic iron overload also causes disturbances in endocrine glands, including the pancreas, pituitary, and thyroid systems. Most of these complications progress slowly and appear in the second decade of life. Thalassemia is the most common genetic disease in the world and has a high prevalence in Iran as well. There are approximately 200,000 homozygous patients and close to 3.75 million carriers in Iran. The highest prevalence of the disease has observed along the margin of the Caspian Sea and Persian Gulf and the provinces of Mazandaran and Fars (1-7). Khuzestan province and the populated city of Ahvaz are of particular interest because of a variety of factors, including the Arab and Bakhtiari tribes and other ethnic groups present, geographic location along the Persian Gulf, tropical weather close to the equator, communication by land and waterways with different regions and numerous wars in this area. This locality can be considered as a large and diverse pool for observing thalassemia and other hemoglobinopathies (8). The number of patients with major thalassemia in Khuzestan province has been estimated to be 2,500 patients (9).

Primary hypothyroidism is one of the most common side effects seen in patients with thalassemia. Cases of hypothyroidism in patients with major thalassemia in different studies, regardless of incidence, have been reported between $13 \%$ and $60 \%$. Prevalence of hypothyroidism in patients with beta thalassemia varied in different studies. The prevalence in Shiraz, Tabriz, and Tehran have been reported, respectively, as $7 \%, 16 \%$, and $7.7 \%$. However, the prevalence has been reported as $21.6 \%$ in Italy (10-13). Increased TSH hormone (thyroid stimulating hormone) with a corresponding decrease in T4, is considered as severe hypothyroidism $(1,14)$. Additional deposits of iron overload in patients with thalassemia are the major cause of subclinical hypothyroidism early in the first phase and eventually leads to obvious hypothyroidism, which is followed by symptoms, including poor growth, obesity, constipation, decreased energy, increased sleep time, and delayed sexual maturity, that require immediate treatment with thyroid hormones (4). Multiple and contradictory results have been reported for the effects of iron overload on thyroid gland functioning and results vary from normal to abnormal (15-17). Thalassemia has a profound impact on the endocrine system function, especially the thyroid and can be studied through its genetic basis and the influence of genetic polymorphisms. Due to the current research contradictions and the high prevalence of thalassemia, regional study of the mechanism of the disease in patients appears to be essential. As a study on this topic has not been completed in the Khuzestan province, the aim of this study is to evaluate the levels of thyroid hormones and TSH in patients with beta-thalassemia in the city of Ahwaz.

\section{Material and Methods}

This case-control study was conducted in Abuzar Hospital in the city of Ahwaz during 2015-2016. The case group included 105 patients with thalassemia that were referred to the treatment center. The control group also consisted of 105 healthy subjects admitted to the same medical center. Before entering the study, possible subjects were checked by researchers and clinicians. Inclusion criteria include the absence of infectious diseases, autoimmune diseases, thyroid and parathyroid glands diseases, acute and chronic gastrointestinal diseases, and that any vaccine or surgery was completed over a month before the test. Obviously, the inclusion criteria originated in the review process. In this study, groups were matched for sex and age and analysis was carried out through the selection of controls appropriate to the case. All subjects were enrolled with informed consent. Blood sampling from the patients was prescribed by a doctor and the required tests were completed after permission from the patients. Recognition and inclusion of patients in the case group was performed based on hemoglobin electrophoresis and all patients were under the medical supervision of a Professor of hematology. None of the patients were receiving sex steroids during the course of the study. Exclusion criteria included a diagnosis of thalassemia minor (mild or moderate), splenectomy, family history of hypothyroidism, thyroid-stimulating drug consumption and the risk of acute diseases, or oncology diseases. From all persons in the case and control groups, fasting blood samples were taken at 8 am for measurement of T3, T4, and TSH. Serum levels of hormones were measured by the Enzyme-Linked Immunosorbent Assay method and with the help of a Stat fax 2100. In order to measure hormone concentrations of T3, T4, and TSH, a kit of Mono Bind was used with a sensitivity $0.12(\mathrm{ng} / \mathrm{ml}), 0.4(\mu \mathrm{g} / \mathrm{dl})$, and $0.078(\mu \mathrm{IU} / \mathrm{ml})$. Data was processed using SPSS 15 (SPSS Inc., Chicago, Illinois, USA) and was analyzed using independent t-test and logistic regression analysis.

\section{Results}

In this study, the case and control groups were the same with regards to age and gender. Regarding gender, the subjects' distributions were similar in both groups and all studied subjects were in the age range of 15-20 years. For comparison, the average of three variable, T3, T4 and TSH from the two groups of case and control and the two independent sample t-test was used according to the normal distribution of data. Serum level of T3 hormone in the case and control groups showed no significant difference ( $p>0.05)$. In this study, serum T4 levels were lower in the 
case group than the control group and the difference was statistically significant $(\mathrm{p}<0.05)$. Serum levels of thyroid stimulating hormone (TSH) were also higher in the treatment group than the control group and the difference was statistically significant $(\mathrm{p}<0.05)$ (Table 1$)$. Logistic regression analysis shows statistically significant differences in serum levels of T4 (OR: 0.58 , with a 95\% confidence level and 0.46 to 0.72 of confidence interval) and TSH (OR: 1.57 , with a $95 \%$ confidence level and 1.22 to 2.01 confidence interval) between the two case and control groups (p $<0.05)$. Interestingly, serum level of T3 in the case and control groups showed no significant difference ( $\mathrm{p}>0.05)$ (Table 2).

Table 1. Comparison of the average of the three variable T3, T4, and TSH in the case and control groups using two independent samples T-test

\begin{tabular}{|l|l|l|l|l|}
\hline Variable & Group & Mean & p-value & Test result \\
\hline T3 & Case & 1.43 & 0.075 & no significant difference \\
& Control & 1.35 & & \\
\hline T4 & Case & 7.23 & 0.00 & significant difference \\
& Control & 8.27 & & \\
\hline TSH & Case & 2.91 & 0.00 & significant difference \\
& Control & 1.92 & & \\
\hline
\end{tabular}

Table 2. Results of the logistic regression test for three variables T3, T4, and TSH in the case and control groups

\begin{tabular}{|l|l|l|l|l|l|l|}
\hline Variable & Wald statistic & p-value & Test result & Exp (B) & B & CI (95\%) \\
\hline T3 & 3.144 & 0.076 & no significant difference & 2.15 & 0.765 & $5.011-0.922$ \\
\hline T4 & 22.85 & 0.00 & significant difference & 0.58 & -0.544 & $0.726-0.465$ \\
\hline TSH & 12.84 & 0.00 & significant difference & 1.57 & 0.454 & $2.018-1.228$ \\
\hline
\end{tabular}

\section{Discussion}

In this study, 105 patients with thalassemia major were compared in terms of the thyroid gland functioning with 105 healthy subjects as controls. The serum T4 levels in patients were lower compared to the control group, while the TSH levels were higher. These results indicate obvious hypothyroidism in patients with thalassemia. The logistic regression analysis showed that serum T4 level in the experimental group was 0.58 , compared to the control group, and serum TSH level in cases is 1.57 times more than the control group. Despite the high ferritin levels in some studies which showed increased body iron, serum levels of pituitary-thyroid were not changed (17, 20, and 21). While in others reports, the direct relationship between serum ferritin and a decrease of the performance of the pituitary-thyroid axis has been demonstrated (22-24). Evaluation the thyroid function is very important due to its tremendous impact on the body's normal growth in infancy and adolescence, as well as participation in many physiological mechanisms. Consequently, due to this vital function, reduced activity of the gland in patients with thalassemia major can be followed by effects, including reduced growth and mental retardation (16). After gonadal failure, hypothyroidism seems to be the most common endocrine disorder in patients with thalassemia major (25). Studies on the thyroid function of patients with thalassemia major have had different results (16). In the Jain study on 25 patients with thalassemia major, the serum levels of T3, T4, and TSH were reported as lower for T3 and T4 and higher for TSH compared to healthy subjects (control group) (17). In two other studies, hypothyroidism was observed in none of the patients with thalassemia major $(20,22)$. These differences in various reports originate from the fact that the prevalence of hypothyroidism varies widely, depending on age, region and type of treatment, which includes the rate of blood collection in a month, chelation therapy, and follow-up intervals of studied patients. The reason for its prevalence involves lack of treatment and poor follow-ups of the disease early in life, which lead to irreversible tissue damage due to excess iron overload $(10,11)$.

Iron poisoning of the thyroid gland may be one of the main causes of hypothyroidism. Also, the effects of iron poisoning on other endocrine glands, such as the gonads, may alter thyroid gland function (26). However, comparing serum ferritin levels in patients with hypothyroidism with the control group showed no logical connection between the current level of serum ferritin and thyroid dysfunction. This is probably due to the fact the serum ferritin levels show the iron overload for the last 3 months, while developing endocrine disorders needs longterm exposure to extra iron $(11,27)$. Therefore, one can conclude that observations of hypothyroidism among these patients may occur due to a chronic increase of iron and its effect on the thyroid gland, followed by a decline in T3 and T4 levels. In addition to the increased toxicity of iron overload in the body, the hypoxia-induced disorder should also be considered. Previous studies have indicated that blood level of hemoglobin in patients with thalassemia is 
approximately 7 to 8 grams per deciliter and a high concentration of 2,3-bisphosphoglycerate and high levels of $\mathrm{HbF}$ may lead to tissue hypoxia, which can have a negative effect on thyroid gland function (24). This finding could also explain the reduced activity of the thyroid gland, or in other words, the hypothyroidism in patients with thalassemia major in whom hypoxia is observed as a result of the disease.

\section{Conclusions}

Hypothyroidism is evident in patients with beta thalassemia major. These findings double the importance of the annual assessment of thyroid function and early diagnosis in patients with beta thalassemia. With regard to the likely effect of iron on this disorder, a blood transfusion program should be prescribed in shorter intervals so that the concentration of blood hemoglobin stays at approximately $11 \mathrm{~g}$ per deciliter. Also, desferal should be regularly injected to prevent hemochromatosis in patients with thalassemia major. Therefore, it is suggested that the iron intake would not exceed the amount of $200 \mathrm{~g}$ per $\mathrm{kg}$ of patient body weight per year to maintain ferritin concentrations at normal levels. While this study was completed at the center of the province, the findings shown here are relevant on a larger scale.

\section{Acknowledgments:}

We thank all laboratory personnel and medical staff of Abuzar hospital who helped us to carry out this project. We also thank Maryam Hadian and Samira Hafezi too for their important help.

\section{Conflict of Interest:}

There is no conflict of interest to be declared.

\section{Authors' contributions:}

All authors contributed to this project and article equally. All authors read and approved the final manuscript.

\section{References:}

1) Zandian Kh, Eshagh Hossani K, Riahi K. A study of prevalence of hypothyroidism in $\beta$ - thalassemia major in Ahvaz Shafa hospital. Scientific Medical Journal of Ahwaz University of Medical Sciences. 2009; 8(62): 298-4.

2) Company F, Rezaei N, Mozafari R. Cardiovascular complications of thalassemia major and thalassemia intermedia. SJKU. 2008; 13(2): 1-9.

3) Chekandi T. Evaluation of thyroid and parathyroid function in patients with thalassemia major. Journal of Birjand University of Medical Sciences. 2004; 11(2): 46-50

4) Company F, Rezaei N, Pourmohmmad B, Gharibi F. Assessment of thyroid dysfunction in patients with Bthalassemia major. SJKU. 2009; 13(4): 37-44.

5) Heydarian S, Jafari R, Karami H. Refractive errors and ocular biometry components in thalassemia major patients. Int Ophthalmol. 2016: 36(2): 267-71. doi: 10.1007/s10792-015-0161-8. PMID: 26646775.

6) Hashemi AS, Ordooei M, Golestan M, Akhavan Ghalibaf M, Mahmoudabadi F, Arefinia M, et al. Hypothyroidism and serum ferritin level in patients with major $B$ thalassemia. Iranian Journal of Pediatric Hematology Oncology. 2011; 1(2): 53-6.

7) Eshragi P, Tamaddoni A, Zarifi K, Mohammadhasani A, Aminzadeh M. Thyroid function in major thalassemia patients: Is it related to height and chelation therapy? Caspian J Intern Med. 2011; 2(1): 18993. PMID: 24024013, PMCID: PMC3766932.

8) Zandian Kh, Kykhai B , Pedram M, Kykhai B , Pedram M, Zandian Kh. Prenatal Diagnosis and Frequency Determination of $\alpha, \beta$-Thelassemia, S, D and C Hemoglobinopathies Globin Mutations among Ahwazian Volunteers. Scientific Medical Journal of Ahwaz University of Medical Sciences. 2006; 5(50): 628-3.

9) Tabatabaie SM. Principles of prevention and treatment of diseases. Nashre Seda Publisher First edition. 123.

10) Shamshirsaz AA, Bekheirnia MR, Kamgar M, Pourzahedgilani N, Bouzari N, Habibzadeh M, et al. Endocrinologic complications in major beta thalassemia: a multicenter study in Tehran. BMC Endocrine disord. 2003; 3: 1472-88.

11) Karamifar H, Shahriari M, Sadjadian N. Prevalence of endocrine complications in beta-thalassaemia major in the Islamic Republic of Iran. East Mediterr Health J. 2003; 9(1-2): 55-60. PMID: 15562733. 
12) Najafipour F, Aliasgarzadeh A, Aghamohamadzadeh N, Bahrami A, Mobasri M, Niafar M, et al. A crosssectional study of metabolic and endocrine complications in beta-thalassemia major. Ann Saudi Med. 2008; 28(5): 361-6. doi: 10.4103/0256-4947.51683. PMID: 18779644.

13) De Sanctis V, De Sanctis E, Ricchieri P, Gubellini E, Gilli G, Gamberini MR. Mild subclinical hypothyroidism in thalassaemia major: prevalence, multigated radionuclide test, clinical and laboratory long-term follow-up study. Pediatr Endocrinol Rev. 2008; 6(1): 174-80. PMID: 19337174.

14) Soliman AT, Al Yafei F, Al-Naimi L, Almarri N, Sabt A, Yassin M, et al. Longitudinal study on thyroid function in patients with thalassemia major: high incidence of central hypothyroidism by 18 years. Indian $\mathrm{J}$ Endocrinol Metab. 2013; 17(6): 1090-5. doi: 10.4103/2230-8210.122635. PMID: 24381890, PMCID: PMC3872691.

15) Mariotti S, Loviselli A, Murenu S, Sau F, Valentino L, Mandas A, et al. High prevalence of thyroid dysfunction in adult patients with $\beta$-thalassemia major submitted to amiodarone treatment. J Endocrinol Invest. 1999; 22(1): 55-63. doi: 10.1007/BF03345479. PMID: 10090138.

16) Rovet J, Alvarez M. Thyroid hormone and attention in congenital hypothyroidism. J Pediatr Endocrinol Metab. 1996; 9(1): 63-6. PMID: 8887135.

17) Jain M, Sinha RS, Chellani H, Anand NK. Assessment of thyroid functions and its role in body growth in thalassemia major. Indian pediatr. 1995; 32(2): 213-9. PMID: 8635784.

18) Marshall WA, Tanner JM. Variation in The Pattern of Pubertal Changes in boys. Arch Dis Child. 1970; 45(239): 13-23. doi: 10.1136/adc.45.239.13. PMID: 5440182, PMCID: PMC2020414.

19) World Health Organization. Indicators for assessing Iodine deficiency disorders and their control programmes. Report of a joint WHO/ UNICEF/ICCIDD Consulation (unpublished document WHO/NUT 193: 1; available on request from the nutrion unit, WHO, Geneva, 1993)

20) Senanayake MP, Suraweera SA, Hubert HD. Thyroid function in thalassaemia major. The Ceylon medical journal. 1999; 44(4): 166-8. PMID: 10895267.

21) Gulati R, Bhatia V, Agarwal SS. Early onset of endocrine abnormalities in beta-thalassemia major in a developing country. J Pediatr Endocrinol Metab. 2000; 13(6): 651-6. doi: 10.1515/JPEM.2000.13.6.651. PMID: 10905390.

22) Jensen CE, Tuck SM, Old J, Morris RW, Yardumian A, De Sanctis V, et al. Incidence of endocrine complications and clinical disease severity related to genotype analysis and iron overload in patients with $\beta$ - thalassaemia. Eur J Haematol. 1997; 59(2): 76-81. doi: 10.1111/j.1600-0609.1997.tb00729.x. PMID: 9293854.

23) Magro S, Puzzonia P, Consarino C, Galati MC, Morgione S, Porcelli D, et al. Hypothyroidism in patients with thalassemia syndromes. Acta haematologica. 1990; 84(2): 72-6. doi: 10.1159/000205032. PMID: 2120889.

24) Agarwal MB, Shah S, Vishwanathan C, Rajadhyaksha G, Bhave AA, Dube SR, et al. Thyroid dysfunction in multi-transfused iron loaded thalassemia patients. Indian pediatrics. 1992; 29(8): 997-102. PMID: 1459722.

25) Multicenter study on prevalence of endocrine complications in thalassemia major. Italian working Group on Endocrine Complications in Non-endocrine Diseases. Clin Endocrinol. 1995; 42(6): 581-6. doi: 10.1111/j.1365-2265.1995.tb02683.x. PMID: 7634497.

26) Berkovitch M, Bistritzer T, Milone SD, Perlman K, Kucharczyk W, Olivieri NF. Iron deposition in the anterior pituitary in homozygous beta-thalassemia: MRI evaluation and correlation with gonadal function. J Pediatr Endocrinol Metab. 2000; 13(2): 179-84. doi: 10.1515/JPEM.2000.13.2.179. PMID: 10711663.

27) Pignatti C, Rugollotto S, De Stefano P, Zhao H, Forni G, Piga A, et al. Survival and complication in patients with beta thalassemia major. Heamatologica. 2004; 89: 1187-93. 